Proceedings of the 2012 Winter Simulation Conference

C. Laroque, J. Himmelspach, R. Pasupathy, O. Rose, and A. M. Uhrmacher, eds.

\title{
COMPLEX AGENT INTERACTIONS IN OPERATIONAL SIMULATIONS FOR AEROSPACE DESIGN
}

\author{
Benjamin Schumann \\ James Scanlan \\ Hans Fangohr \\ University of Southampton \\ University Road \\ SO17 1BJ Southampton, UK
}

\begin{abstract}
Product complexity in the aerospace industry has grown fast while design procedures and techniques did not keep pace. Product life cycle implications are largely neglected during the early design phase. Also, aerospace designers fail to optimize products for the intended operational environment. This study shows how a design, simulated within its anticipated operational environment, can inform about critical design parameters, thereby creating a more targeted design improving the chance of commercial success. An agent-based operational simulation for civil Unmanned Aerial Vehicles conducting maritime Search-andRescue missions is used to design and optimize aircrafts. Agent interactions with their environment over the product life-cycle are shown to lead to unexpected model outputs. Unique insights into the optimal design are gained by analysis of the operational performance of the aircraft within its simulated environment.
\end{abstract}

\section{INTRODUCTION}

Ever growing product complexity strains organizational decision-making structures established decades ago. Companies still use relatively traditional processes and design tools that are guided by a chief engineer whose experience and intuition lead him to design decisions based on analysis from different disciplines (Bond and Ricci 1992). However, contemporary product complexity and project scales render this method more and more obsolete and chief engineers are forced to negotiate consensus-driven decisions by large number of specialist teams. Decision quality hinges on communication and information clarity. Even with ideal information quality, decision trade-offs are often focused on short-term success, neglecting operational information on long-term cost and environmental impact.

Most acutely, this lack is observed during the early design phase where design decisions weigh most heavily (Raj 1998). Early development is limited by budget and time constraints as much as by lacking knowledge about how the product is best designed for its intended market. Whereas designers routinely analyze design changes with regards to performance changes, the link to operational variations is not commonly possible. In other words, it is easy to answer "How much faster can I fly by reducing the wing span by 10\%?" but much more difficult to investigate "How much money do I save over 5 years operating an aircraft with a $10 \%$ smaller wing span"? An operational simulation can support such queries by linking design quality and performance to operational constraints. One can argue that the latter question is of superior importance because it is meaningful to management and customers. The first question is only important for other engineers. Currently, aerospace companies have a good understanding of answering the former question while struggling to even approach the latter question with confidence. These observations led to the work reported here, which is carried out as part of the DECODE (Decision Environment for COmplex Design Evaluation) research project. 


\section{Schumann, Scanlan, and Fangohr}

DECODE aims to provide a holistic design decision support environment. Uniquely, DECODE provides active design exploration and optimization of system level trade-offs between performance, cost and operational influences. The goal, beside others, is to enable designers to understand the link between design decisions and life-cycle performance. This vision is realized by designing a number of real Unmanned Aerial Vehicles (UAVs) using an evolving DECODE toolbox and comparing their performance. This toolbox links a number of traditional design software (CAD, CFD, structural analysis) with an operational simulation and a value model such that a new design work flow can be explored. The UAVs in this project are designed for maritime Search-and-Rescue (SAR) support missions. It is beyond the scope of this paper to provide a detailed description of the DECODE toolbox. Further information can be found in Schumann, Scanlan, and Takeda (2011b) and (Gorissen et al. 2012). Moreover, Schumann, Scanlan, and Takeda (2011a) provides a case study trying to answer the (managerial) question "Is it better to use many cheap small UAVs or one large expensive UAV?".

A core concept of DECODE is the use of an operational simulation to design and optimize UAVs for their intended missions. In the traditional sense, operational simulations are highly detailed and realistic models of factory floors that support short-term "live" production planning (Andersson and Olsson 1998). During the last two decades, usage has spread to transportation management (Demitz, Hübschen, and Albrecht 2010) and supply chain simulation. DECODE extends the use of an operational simulation to aerospace design.

Despite the obvious advantages of using an operational simulation to improve design, the uncertainties of modeling an operational environment for aerospace products are much greater than those of a well-known factory floor. The time frames involved are several magnitudes larger because the simulation models a future product in its future environment, often a decade or more in advance for aerospace products (Kirby 2001). These uncertainties grow exponentially with product design time. Civil UAVs currently have a much shorter design times. Therefore, designing UAVs offers a unique opportunity to explore operational simulations for larger aerospace products. Further uncertainty comes from outside environmental factors such as business competitors or market changes. Moreover, interactions of the product with its environment must be anticipated, often lacking trustworthy data. Consequently, the model described here cannot hope to give an accurate estimate of performance of the final product. Instead, it is envisaged to guide designers and support their decisions by informing about trends, theoretical optimums and unforeseen consequences of design decisions. However, this must not distract conducting sensitivity analysis for inferior data, otherwise even such a "proof-of-concept" tool can be misleading.

Immersing a design into its intended (virtual) environment adds complexity to the design process. Even for relatively simple designs, unforeseen outputs can radically change the final product. This paper studies how the use of an operational simulation for the design of a maritime SAR UAV creates unexpected results. Two case studies explaining those counter-intuitive outputs are presented. The rest of the paper is structured as follows: Section 2 details the operational simulation developed for DECODE. It presents agent behaviors, outputs and the specific scenario for the case studies. Section 3 shows how results for this research were obtained. Section 4 explains two case studies taking into account agent interactions and environmental constraints. Subsequently, Section 5 discusses the implications of the results for DECODE and the wider simulation community.

\section{THE OPERATIONAL SIMULATION}

This section introduces the operational simulation used in this study.

\subsection{Software Selection}

The software of choice is AnyLogic ${ }^{\complement}$ (Version 6.7), a software tool developed by XJ Technologies ${ }^{\complement}$. AnyLogic offers a true agent-based framework embedded in a classical discrete-event environment. The 


\section{Schumann, Scanlan, and Fangohr}

Java-based software allows multi-core evaluation of design points, enabling exploration of large design spaces and long life-cycles.

\subsection{Model Scenario}

The case studies in this paper are conducted within a maritime Search-and-Rescue (SAR) scenario. The effectiveness of locating people and vessels out at sea in possibly life-threatening situations can be significantly improved by additional camera vision from the sky and has been identified as a key application area for civil UAVs (Herrick 2000). Practical matters such as certification, safety and camera capabilities will not be discussed here because the research is concerned with proof-of-concept capabilities at this stage. The specific mission scenario for DECODE is based around the south coast of the UK, as in Figure 1.

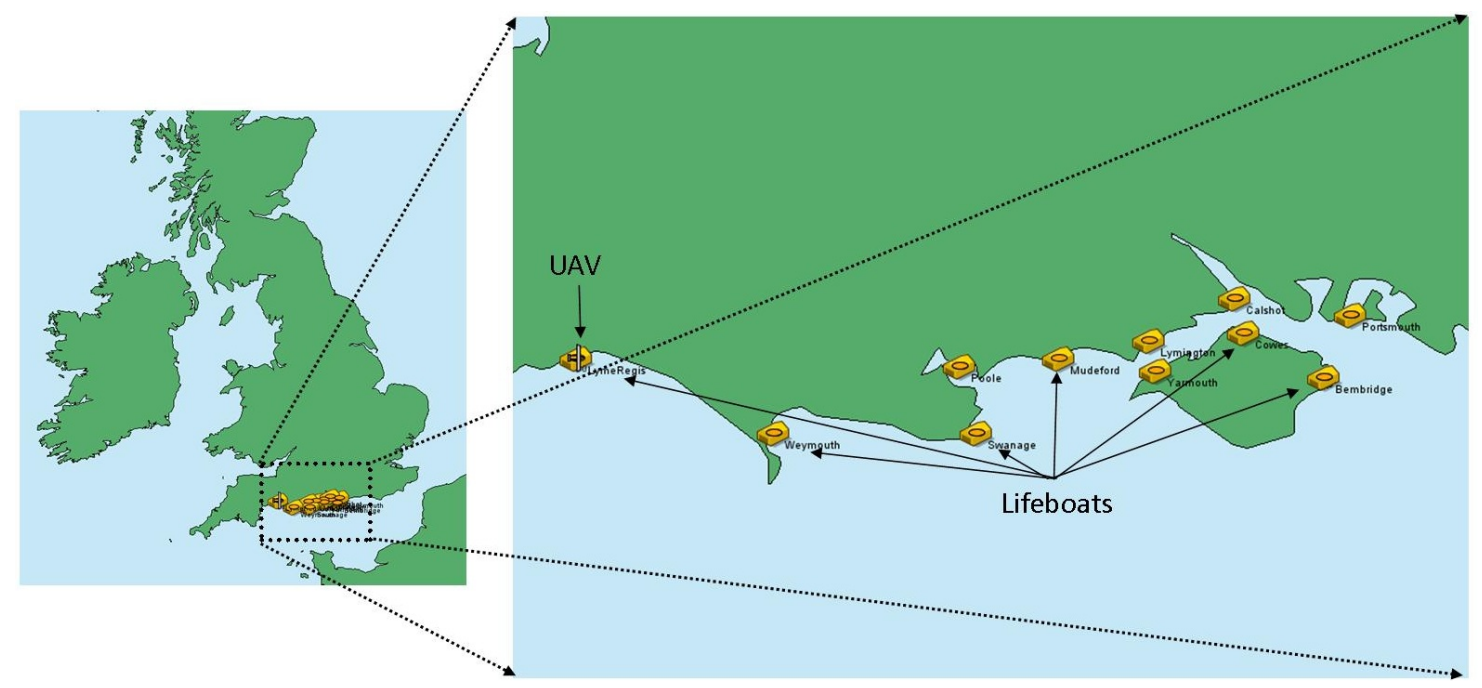

Figure 1: The SAR scenario used in this paper.

In reality, there are 11 rescue stations equipped with various types of lifeboats in this area, covering an area of approximately 7800 square kilometers. Once an incident is reported to the authorities, they dispatch one or all lifeboats of the nearest station. Currently, no UAVs are part of the SAR-activities in the region. This simulation will model the impact of introducing UAVs.

In the simulation model, rescue stations and lifeboats are represented through agents. Lifeboat agents vary in dash and search speeds according to their type. Lifeboats are distributed among the stations as in reality. They dispatch upon receiving an emergency signal from incident-agents and start a search operation (see Section 2.4).

Each rescue station has a given number of incidents occurring each year based on historic values (see Figure 2). This value is used to calculate random incident occurrence events. Once an incident occurs, its distance to the rescue station is drawn randomly from the distribution seen in Figure 3. Additionally, a random bearing is assigned. If this position is not on any landmass, the incident is created and other agents are notified about it.

The simulation runtime is set to a five-year period to cover seasonal variations in incident appearance and weather. Moreover, long-term effects such as UAV crashes can be observed and analyzed.

\subsection{The UAV}

Only the most westward station "Lyme Regis" houses one UAV. However, this UAV will be called for search support for any incident along the south coast to force the UAV to cover long distances before it can actually support searches. This is desirable to be able to distinguish very similar designs in the case 


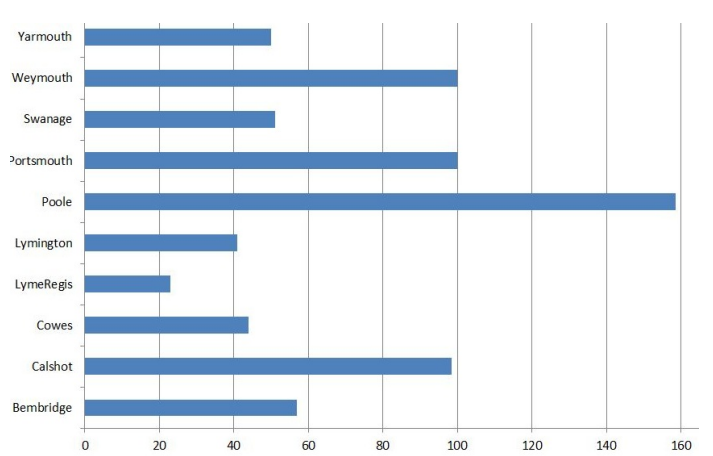

Figure 2: Incidents per year for every station. Source: RNLI

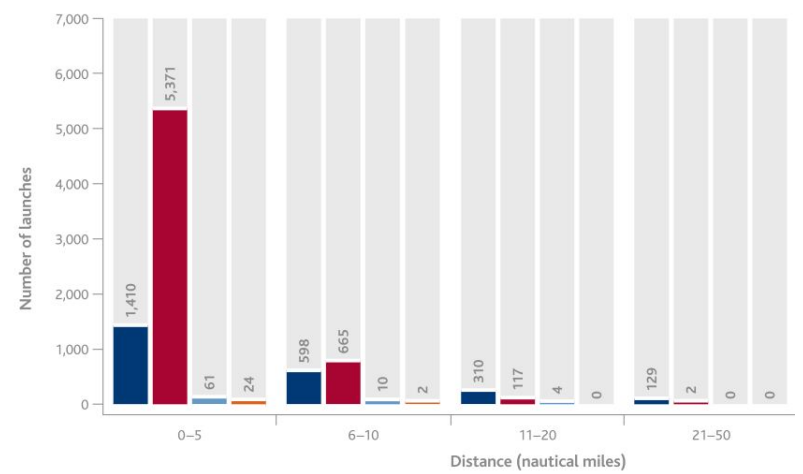

Figure 3: Distance-to-station distribution for incidents. Source: (Vlasto 2009; Norris 2009)

study outputs. The UAV used in this study is propelled by a twin boom "pusher" propeller and its tail has an inverted v-tail to reduce drag (Figure 4). The UAV weighs between 18 and 24 kilograms (depending

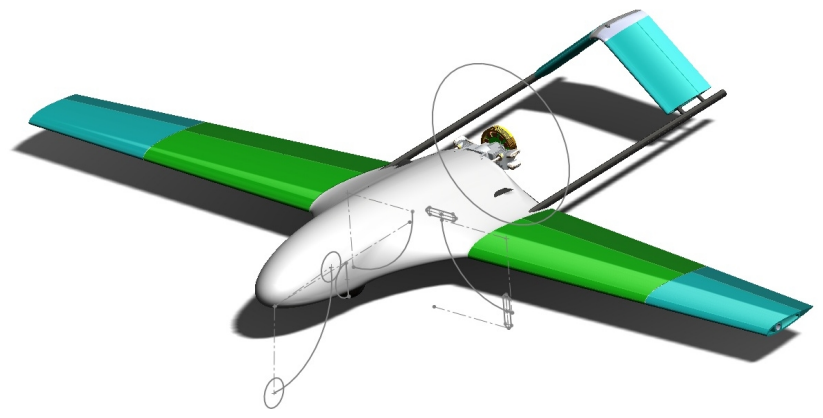

Figure 4: The UAV configuration used: "twin-boom pusher with inverted v-tail."

on the design choices) and spans approximately 4 meters.

\subsection{Search Description}

Once an incident occurs, lifeboats and the UAV dash out to the "initial search position", a point somewhere near the incident symbolizing the last known position or best position guess. Upon arrival, the agents start an "expanding square pattern" search, an internationally agreed search pattern suitable for incidents with relatively good knowledge of position (IAMSAR 2007). This pattern can be seen in Figure 5.

The pattern depends on the size of the scanned area which depends on camera capability, cruise height and weather. Generally, the scanned area is larger for heavier (i.e. better) on-board camera systems, higher cruise heights, larger incidents (lost yachts are easier to spot than a drowning "head in the water") and better weather conditions. The larger the scanned area, the sooner the UAV will find a casualty. Lifeboats follow the same pattern but their pattern scan sizes depends on the size of the crew on board, weather and the type of the incident only.

In reality, image analysis software on board the UAV constantly processes the images taken. It is tuned such that it recognizes the incident type. In such a case, the image in question will be sent to a manned ground station where an operator can agree or disagree with the software's findings. If he agrees, the nearest lifeboat will be sent to the UAV position and the UAV returns home. Otherwise, the UAV is asked to reduce height and cross the spot in question again to take a close-up photo for the operator. In 
Schumann, Scanlan, and Fangohr

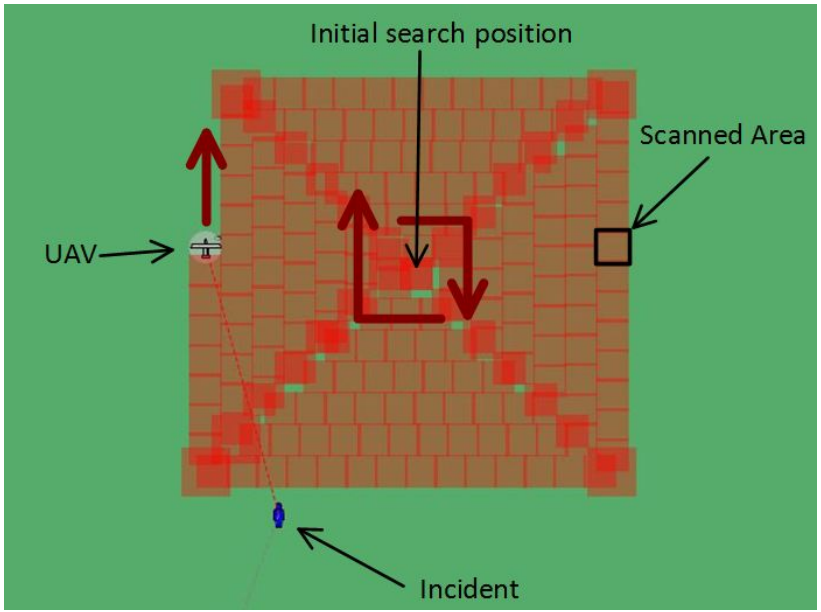

Figure 5: A typical search procedure.

this simulation model, a simple two-minute delay imitates this case, i.e. the UAV freezes its expanding search pattern for two minutes.

In order to analyze differences between UAVs, design decisions must exert an influence upon the performance output of the operational simulation. One way to achieve this is by correlating the search performance of a UAV with its payload mass. Payload mass correlates with the quality of the camera system on board, i.e. a UAV that is designed to carry more payload can house a heavier camera system. This model assumes that a heavier camera system has better quality in terms of spotting incidents. According to Westall et al. (2008), there are two influences that must be simulated when recreating camera-driven search for humans: the False Alarm Rate (FAR) and the Missed Detection Rate (MDR).

\subsubsection{False Alarm Rate}

When a UAV searches for a missing person, it may occasionally happen that the image analysis software carried on board mistakes a wave crest or any other item floating on the sea for the head of a person or for the life raft in question. To model this, we define the False Alarm Rate (FAR) as the fraction of images that will report a false positive, i.e. those that will report an incident where there is none.

During each image capture, a random decision to spot a false incident will be taken based on the FAR. Therefore, a low FAR leads to quicker spotting of the actual incident as each false positive causes a 2-minute delay for subsequent investigations as outlined above. Based on observations from Westall et al. (2008) and engineering judgment, the FAR for persons floating in water varies with payload mass as in Figure 6. Note that UAVs designed by DECODE will not exceed the payload range between 0.5 and 7 kilograms.

\subsubsection{Missed Detection Rate}

Once the UAV actually crosses the incident in question, it can either recognize it and notify the human operator at the ground station or it can pass the incident without noticing. Therefore we introduce the Missed Detection Rate (MDR) which indicates how often, on average, per incident crossed the UAV will not notice the incident and thus not notify the authorities.

Again, the MDR is assumed to be correlated with payload mass such that a heavy-payload UAV spots an incident with a higher probability. A possible model for this is shown in Figure 7.

If the UAV fails to identify the incident, it will continue its search in an ever-expanding square pattern until it runs out of fuel. The UAV will return to refuel at its base station. Subsequently, it will launch again to find the incident, possibly crossing it a second time. If, during an expanding-square pattern, the 


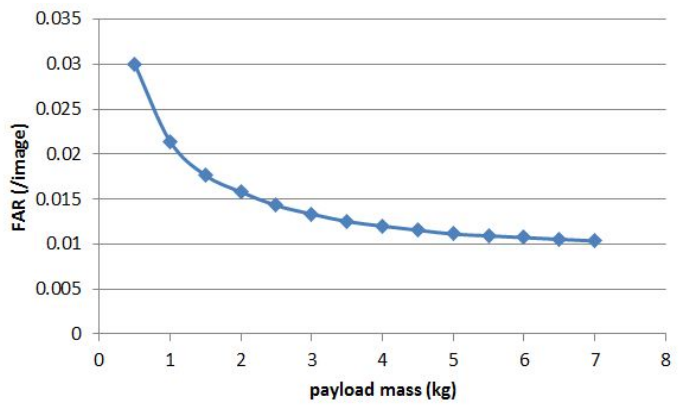

Figure 6: False alarm rate (FAR) versus payload.

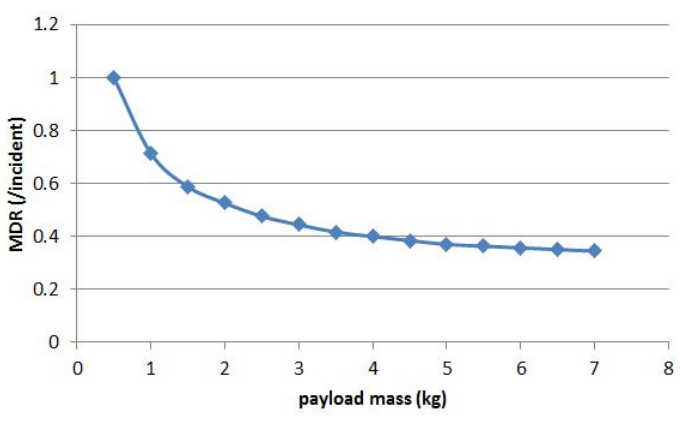

Figure 7: Missed detection rate (MDR) versus payload.

UAV covered a search area larger than 40 by 40 kilometers, it will initiate a new pattern at a different start location. This imitates real search behavior where a maximum search area is defined and covered multiple times if necessary. The UAV only makes an impact on search operations if it finds the incident the first time. Otherwise, either other lifeboats searching together with the UAV will have found the incident or too much time will have passed to rescue the incident alive.

\subsection{Simulation Outputs}

As discussed in Section 1, the operational simulation informs the designer about the performance of his design as part of a suite of softwares. Hence, the output of the simulation is fed into subsequent analysis programs. The outputs must be unambiguous, clear and informative. Three sets of outputs have been identified to be able to calculate the "value" of a design easily: Scenario outputs, UAV outputs and performance outputs.

Scenario outputs relate to quantifiable data from agents that are part of the wider scenario selected by the user. They are expendable according to user needs, i.e. if a scenario includes other agents such as helicopters, appropriate outputs will be generated:

- Number Of Lifeboats Used: How many lifeboats were used in the scenario?

- Lifeboat Total Use Time: How much time did all lifeboat agents spent out at sea?

- Lifeboat Total Calls: How often where lifeboats called out for searches?

UAV outputs are similar in nature but provide more detailed information for the value calculation:

- Number of UAVs Used: How many UAVs were bought in total, including crashed UAVs.

- UAV Number of Takeoffs: How often did UAVs take off? This includes take-offs after refuel.

- UAV Total Flight Time: How much time did all UAVs spend in the air?

- UAV Fuel Used: How much fuel did all UAVs burn in total?

- UAV Number of Maintenance Operations: How many maintenance operations had to be carried out?

- UAV Maintenance Man Time: How much time was required for all maintenance operations?

Performance outputs measure how well a mission goal is achieved. In the context of SAR, mission success hinges upon finding people quickly and alive:

- Number of Saved Lives: How many lives were saved in total?

- Average Incident Waiting Time: How long did incidents have to wait on average? 


\section{Schumann, Scanlan, and Fangohr}

It is possible to calculate a "value" from a specific UAV design using the outputs above. More importantly, the designer must be able to compare different designs and explore design spaces. For this, three-dimensional surface plots can be helpful. However, surfaces must be smooth to be able to deduct trends and find optimal designs. Otherwise, neither automated design optimization nor manual design analysis is feasible.

\section{METHOD}

Analysis is based on surface plots that compare 60 different UAV designs by their variation in permissible payload and landing speed (see sample plot in Figure 8). Every design is based on the same UAV configuration (Figure 4).

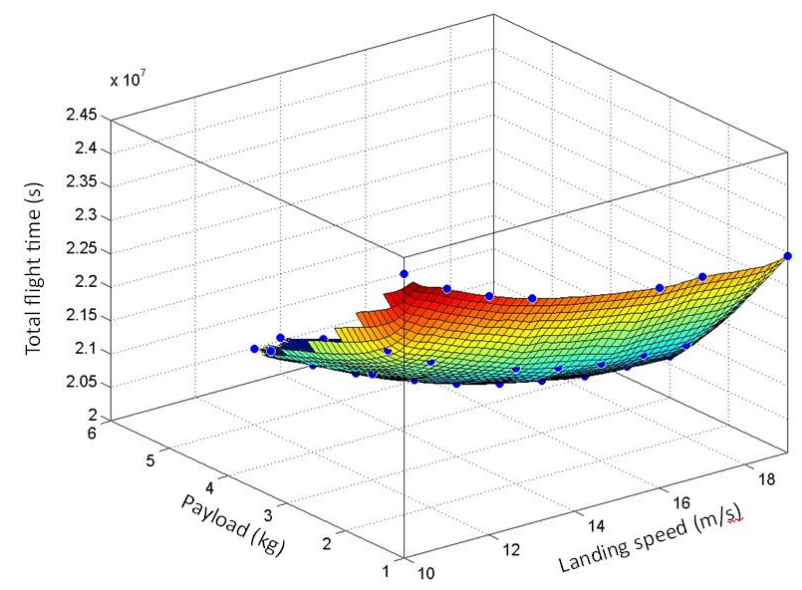

Figure 8: A sample surface plot.

Increasing the permissible payload changes the UAV design such that larger wings are necessary and maximum flight speed reduce slightly. However, a better camera can be used. Increasing the landing speed acts as a design variable that correlates with the general speed of the UAV. If landing speeds can be higher, wing size can be reduced because less lift is required during landing. This, in turn, allows higher flight speeds because smaller wings create less drag. Also, overall fuel consumption is reduced.

In DECODE, the designer creates a UAV design and forwards design parameters to the operational simulation. These parameters include payload, landing speed, fuel tank capacity and a number of aerodynamic coefficients to calculate flight performance for that specific design. Each design is simulated over the fiveyear period for 150 replications. Results are averaged and represented as dots in the surface charts. Confidence is consistently above $95 \%$. Random variables include:

- False alarm: Based on the FAR (see Section 2.4.1), does the UAV spot a false incident during taking a picture or not?

- Missed detection: Based on the MDR (see Section 2.4.2), does the UAV spot the true incident while actually overflying it?

- Time to failure: Each UAV component has a random time-to-failure drawn from a Weibull-distribution with given shape and scale parameters. It indicates after how many flight hours the part will fail. Estimates are based on industry standards for similar components.

- In-flight crashes: If a component fails in-flight before its scheduled maintenance, there is a given probability that the whole aircraft crashes.Estimates are based on industry standards for similar components.

- Landing crashes: Based on the kinetic energy (i.e., landing speed and weight) of the UAV on landing, a crash probability is used to determine if the UAV crashes on landing. 
Surface plots are created for all simulation outputs defined in Section 2.5.

\section{RESULTS AND ANALYSIS}

The following two sections introduce and explain typical user cases where an operational simulation creates new and unexpected insights into the design. Results are based on the scenario setup described above.

\subsection{How UAV Design Influences the Number of Takeoffs}

Figure 9 shows the variation in the total number of takeoffs the UAV conducts over the five-year period of the simulation. It can be seen that a generally faster UAV (higher landing speed) leads to a modest

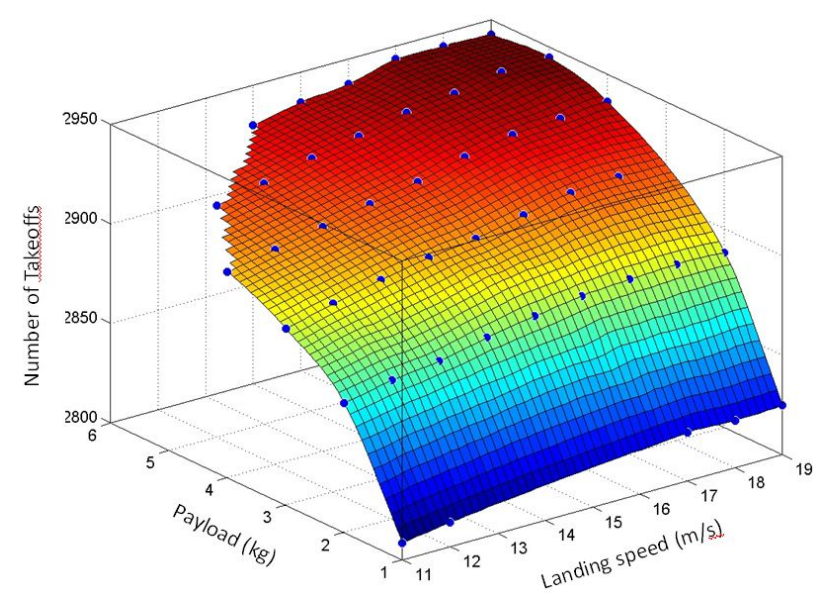

Figure 9: The total number of takeoffs over five years as a function of payload and landing speed.

increase in the number of takeoffs whereas an increase in permissible payload leads to a strong increase in the number of takeoffs. The absolute values indicate that a UAV has to takeoff between 2800 and 2950 times over five years in order to support SAR around the south coast of the UK (equates to an average of 1.5 take-offs per day).

Intuitively, an aircraft engineer would expect no influence of the design upon the number of takeoffs. Surely, a faster UAV would have to fly as many missions as a slower UAV? Why would a heavier UAV with a better camera need to take off more often than a lighter UAV?

However, designing for a higher landing speed allows the UAV to generally fly faster. Therefore, a faster UAV returns home from a mission earlier than a slower UAV. Occasionally, it is notified of a subsequent incident that needs to be searched for and launches for this subsequent mission. The slower $\mathrm{UAV}$, on the other hand, returns to its base later by which time the subsequent incident has been found already by other vessels. Therefore, faster UAVs generate more takeoffs than slower UAVs.

Similarly, more payload means that the UAV spots incidents quicker on average (it misses them less often on overflight due to the MDR and has less in-flight investigations due to the FAR). The same argument holds in this case: the heavier UAV sometimes returns home earlier and may start a subsequent mission.

\subsection{Saving More Lives Does Not Have to Cost More Fuel}

Figure 10 shows that a generally faster UAV (higher landing speed) saves more lives. Similarly but at a greater rate, a UAV with more payload saves more lives. Figure 11 shows that having a generally faster UAV leads to a reduced total fuel consumption. Using a heavier UAV with better camera equipment requires a modest amount of additional fuel. 


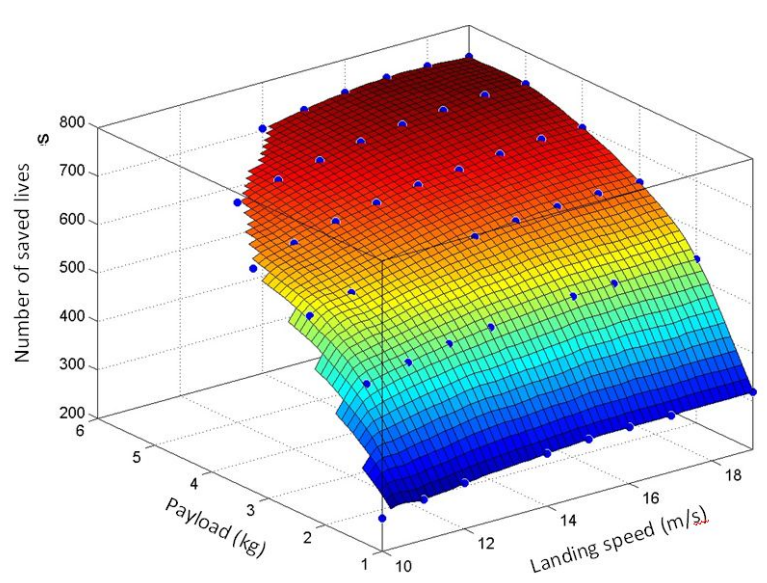

Figure 10: the number of saved lives over five years as a function of payload and landing speed.

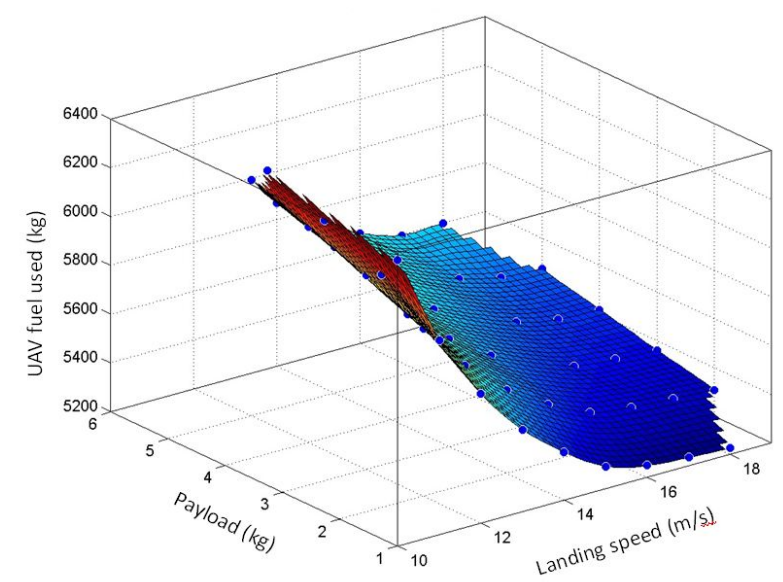

Figure 11: the total fuel used over five years as a function of payload and landing speed.

A higher landing speed allows the designer to employ smaller wings which save weight and reduce drag during flight. This reduces the specific fuel consumption and explains why higher landing speeds result in less fuel used. Simultaneously, higher landing speeds allow the UAV to fly faster which results in more incidents being found earlier and alive.

A higher payload increases the fuel burn because the aircraft becomes heavier and requires more power (i.e. fuel) during flight. In addition, increasing the payload while keeping the landing speed constant requires larger wings to produce the required lift. This, in turn, produces more drag which must be overcome by burning more fuel. However, there is a balancing effect to this: the operational simulation also evaluates the effect of a higher payload, namely that UAVs find incidents earlier. The UAVs have fewer false alarms (FAR) and miss the incident less often upon crossing it (MDR). Therefore, the UAV spends significantly less time in the air, leading to a reduction in fuel consumption while increasing the number of incidents found alive. The simulation combines these two effects and informs the designer that the former is more acute in the chosen scenario.

Intuitively, an engineer would expect that in order to obtain better performance (i.e. save more lives) higher costs (i.e. burn more fuel) are inevitable. At some point, it becomes unfeasible to increase performance further because costs rise exponentially. However, comparing Figure 10 and 11 reveals that this is only partly true for this scenario. Saving the maximum amount of lives does not require the maximum amount of fuel. This is a key finding because the reason to employ UAVs in SAR is to save as many lives as possible.

Note that the reduction in fuel burn is only valid within the current design regime of medium flight speeds and aircraft sizes. At some point, drag would increase and fuel usage would rise again when flying faster.

\section{DISCUSSION}

It has been shown how an operational simulation can reveal and explain unexpected behavior to the designer. The following lists some lessons that a designer could learn from the results.

The first case study above informed the designer that a better or faster UAV (more payload or higher landing speeds) will accumulate more takeoffs. This must be taken into account during part design of the undercarriage which must become more robust. This, in principle, will increase the overall weight of the aircraft which will reduce general flight speeds again. This would cause a reduction in takeoffs. It is beyond the scope of this paper to investigate the quantitative nature of this negative feedback loop. 
The second case study demonstrated that the UAV should have a large payload and a large landing speed. However, this will mean that more UAVs crash during landing because their kinetic energy grows linearly with extra weight and exponentially with higher speeds. Either the designer chooses to strengthen the undercarriage or a higher number of landing crashes (i.e. new UAV purchase) is deemed acceptable. For the case study above, the number of UAVs crashed and re-purchased is shown in Figure 12. It is vital

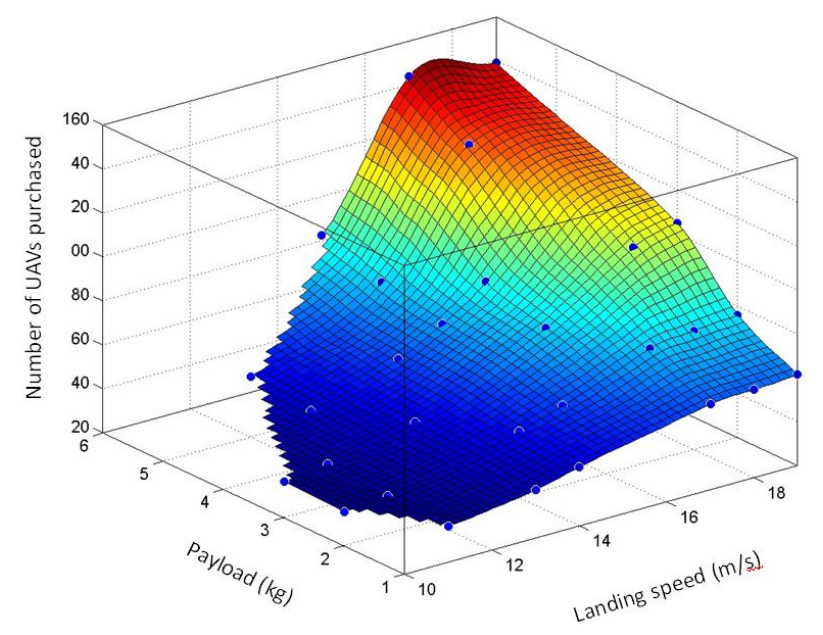

Figure 12: The total number of UAVs purchased as a function of payload and landing speed.

to find a trade-off between better performance and the number of UAVs purchased in total. This is where the value function becomes useful: it weighs product performance factors (mass, speed) against operational performance (number of saved lives, number of crashed UAVs) and returns a simple (monetary) number to help decide if it is better to use a well-equipped UAV that often crashes or an inferior UAV that lasts longer. Results are currently analyzed and will be published separately.

The operational simulation presented here is a proof-of-concept tool implemented into the UAV design suite developed by the DECODE-team. We note that absolute values of the outputs have significant uncertainty. A wide range of assumptions, both data- and procedure-wise create uncertainty. In an industry setting, this level of uncertainty must be overcome by rigorous data mining and model building. However, this additional work would not change the trends of the output plots significantly. It has been shown that much can be learned from these trends alone and they can be used to steer design decisions where rigorous model building is not possible.

The results presented here mean that UAV designs can be tailored towards a specific desired operational scenario. For the DECODE project, this means that it is possible to optimize the design for its intended operational environment to a degree that has not been achieved before. The simulation will be used to analyze a range of design configurations (not just the one used for this study) and their design spaces. It will be possible to choose the best configuration and optimize its design for best overall value.

For the wider modeling community, there are two new developments exemplified here: On the one hand, an optimum design can be chosen based on a global value calculation that takes into account a number of life-cycle considerations (fuel usage, maintenance issues, crashes...). On the other hand, the operational scenario itself can be optimized: Is it better to use many small and cheap UAVs in a swarm or one large, highly capable but expensive UAV? Is it better to station UAVs at every lifeboat station or just at strategic points of interest? It is this combination of information that can distinguish a good from an outstanding design. Although discussion has been limited to UAVs in this paper, the general design approach can be extended to many other areas such as electric cars. 


\section{CONCLUSION}

This paper introduced the usage of an agent-based operational simulation to support design of UAVs for SAR missions. It has been shown that an operational simulation can be used to create novel knowledge about the behavior of an aerospace design in its intended operational environment. This knowledge can challenge engineering intuition because complex agent interactions over the life-cycle of the product create unexpected performance outputs. It has been shown that it is possible to explain such results by consulting the simulation operation methods.

This capability is useful to design and optimize a UAV for SAR missions. Extending the operational simulation will enable designing UAVs for other applications such as pipe-line monitoring, volcano ash analysis or forest fire detection.

\section{ACKNOWLEDGMENTS}

The authors would like to thank the EPSRC funding this work with the Doctoral Training Center grant no. EP/G03690X/1.

\section{REFERENCES}

Andersson, M., and G. Olsson. 1998, December. "A simulation based decision support approach for operational capacity planning in a customer order driven assembly line". In Proceedings of the 1998 Winter Simulation Conference, edited by D. J. Medeiros, E. F. Watson, J. S. Carson, and M. S. Manivannan, 935-941. Piscataway, New Jersey: Institute of Electrical and Electronics Engineers, Inc.

Bond, A. H., and R. J. Ricci. 1992. "Cooperation in Aircraft Design". Research in Engineering Design 4:115130.

Demitz, J., C. Hübschen, and C. Albrecht. 2010. Timetable planning and information quality, Volume 1, Chapter A, 11-25. WIT Press.

Gorissen, D., E. Quaranta, M. Ferraro, B. Schumann, J. van Schaik, A. Keane, and J. Scanlan. 2012. "A Decision Environment for Complex Design Evaluation”. AIAA Journal. Under review.

Herrick, K. 2000. "Development of the Unmanned Aerial Vehicle Market: Forecasts and Trends". Air \& Space Europe 2 (2): 25-27.

IAMSAR 2007. "International Aeronautical and Maritime Search and Rescue Manual Volume III". Technical report, International Maritime Organisation, 4 Albert Embankment, London, SE1 7SR, UK.

Kirby, M. R. 2001, March. A Methodology for Technology Identification, Evaluation and Selection in Conceptual and Preliminary Aircraft Design. Phd-thesis, Georgia Institute of Technology.

Norris, R. 2009. "Helicopter Sortie Reports". Lee-on-Solent, Maritime Coastguard Agency.

Raj, P. 1998. "Aircraft Design in the 21 st Century: Implications for Design Methods". AIAA Journal (AIAA 98-2895).

Schumann, B., J. Scanlan, and K. Takeda. 2011a, March. "Evaluating Design Decisions in Real-Time Using Operations Modelling”. In Air Transport and Operations Symposium 2011 (ATOS), edited by R. Curran and S. C. Santema, 1-10. Delft, the Netherlands: Delft University of Technology.

Schumann, B., J. Scanlan, and K. Takeda. 2011b. "A Generic Operational Simulation for Early Design Civil Unmanned Aerial Vehicles". In SIMUL2011: The Third International Conference on Advances in System Simulation, edited by E. Williams and P. Lorenz, 1-4. Barcelona, Spain: IARIA. ISBN: 978-1-61208-169-4.

Vlasto, M. 2005-2009. "RNLI Annual Operational Statistics Report". Technical report, Royal National Lifeboat Institution, West Quay Road, Poole, Dorset, BH15 1HZ, UK.

Westall, P., J. J. Ford, P. J. O'Shea, and S. Hrabar. 2008, 1-3 December 2008. "Evaluation of Maritime Vision Techniques for Aerial Search of Humans in Maritime Environments". In Digital Image Computing: Techniques and Applications (DICTA), edited by A. Robles-Kelly, 176-183. Canberra, Australia: IEEE. 


\section{AUTHOR BIOGRAPHIES}

BENJAMIN SCHUMANN studied Aerospace Engineering (BEng) and Complex Systems Simulation (MSc) at the University of Southampton, UK. Currently he studies for a PhD with the Institute for Complex Systems Simulation. His research interests focus on improving the design process of aerospace products by using operational simulations. His email address is Benjamin.Schumann@soton.ac.uk and his website can be found at http://cmg.soton.ac.uk/people/bs804/.

JIM SCANLAN received a materials science degree from Manchester University in 1977. He then spent over 12 years in the aerospace industry in a variety of roles, his final post being Head of Manufacturing Research at BAe regional aircraft. Whilst at BAe, Jim studied for an MSc at Salford University in aerospace design, and was sponsored by British Aerospace to study for a year at Cranfield University on a manufacturing management fellowship program. Jim joined the University of the West of England in 1990 and completed a PhD in 1995 in computer modeling of the Aerospace Design Process. Jim was appointed to Reader in 2004. In September 2004 Jim accepted a post at Southampton University as director of design within the Computational Engineering Design Center (CEDC). Jim manages a number of research programs sponsored by BAE systems, Airbus, Rolls-Royce and the EPSRC. He has a particular interest in Design, Logistics, Simulation and Optimization of organizations. His email address is J.P.Scanlan@soton.ac.uk.

HANS FANGOHR is a Professor of Computational Modeling in the School of Engineering Sciences at the University of Southampton. He received his undergraduate degree "Diplomphysiker" in physics from the University of Hamburg (Germany) and completed his $\mathrm{PhD}$ studies in the High Performance Computing Group at the department of Electronics and Computer Science in Southampton. He is leading the Computational Modeling Group at the University of Southampton. He is the co-director of the Institute for Complex Systems Simulations and is directing the learning and teaching of scientific computation in the Institute's Doctoral Training Center. His email address is Fangohr@soton.ac.uk and his web page is http://www.soton.ac.uk/ fangohr/. 